\title{
Do You Want an Inspirational Career? Choose Nuclear Medicine
}

\author{
Steven M. Larson \\ Memorial Sloan Kettering Cancer Center, New York, New York
}

Know Thyself.

—Delphic maxim

The Unexamined Life is Not Worth Living.

-Attributed to Socrates

All specialties of medicine require a continuous stream of skilled trainees in order to renew and grow. Over the $75 \mathrm{y}$ of its existence, the field of nuclear medicine has rejuvenated itself repeatedly by welcoming waves of diverse trainees with adventuresome spirits who recognize the promise of the tracer principle for scientific discovery, clinical diagnosis, and targeted radionuclide therapy. Most recently, the rapid development of molecular imaging for precision medicine has made the field attractive to trainees seeking to combine expertise in nuclear medicine with high-level abilities in cross-sectional imaging. Also, substantial advances in therapeutic index achieved with modern targeted radionuclide therapy, combined with quantitative PET and SPECT imaging for dosimetry, have opened up exciting opportunities for radiotheranostics, ushering in a distinct group of trainees who want to combine nuclear medicine expertise with therapy including patient management. The training programs of the future will need to directly address the special needs of these diagnosticians and therapists. A subset of these groups will likely seek additional training in clinical or research fellowships to deepen their expertise in molecular imaging or targeted therapy. This enhanced training will increase the likelihood for an impactful career, whether in research, clinical care, or both. If we are truly going to attract the best and brightest students to nuclear medicine, leaders and mentors in the field must educate themselves in both aspects of nuclear medicine so we can fully capitalize on the opportunities to improve patient care with radionuclides.

J Nucl Med 2019; 60:25S-28S

DOI: 10.2967/jnumed.118.220574

\section{I}

was asked to write on the topic "Rejuvenating an Entire Field: How Can We Attract Young People to NM for Training for a Theranostic Career?" With the kind indulgence of the editors, I changed the title to focus slightly more on the trainees, who should be in the spotlight. Still, I do have 2 groups in mind as the target audience: ( 1 ) young medical professionals in early training who have yet to choose a specialty; and (2) leaders/mentors who are responsible for setting nuclear medicine training standards

Received Apr. 23, 2019; revision accepted Jun. 18, 2019.

For correspondence or reprints contact: Steven M. Larson, Memorial Sloan Kettering Cancer Center, 1275 York Ave., S-212, New York, NY 10021.

E-mail: larsons@mskcc.org

COPYRIGHT (C) 2019 by the Society of Nuclear Medicine and Molecular Imaging.

and overseeing training programs. When I say leaders and mentors, I mean program directors, Administrative Chiefs of Nuclear Medicine and Radiology, Directors of the Boards of Nuclear Medicine and Radiology, and other stakeholders in professional organizations such as the Radiological Society of North America, Society of Nuclear Medicine and Molecular Imaging, and European Association of Nuclear Medicine. Especially in the United States, where most nuclear medicine groups are considered part of Radiology, the Chairman or equivalent has considerable influence over the training and educational opportunities in the field of nuclear medicine.

I will offer my perspective on this topic of inspirational career through the lens of my own personal experience, bearing in mind the crucial precept for trainees: "Know thyself." For the leaders/mentors, I am reminded that our role is rightly honored in our society - a high calling with the likelihood of great satisfaction. But I am reminded also of a teacher's special responsibilities; we must ensure that the benefit to our trainees is our highest priority, second only to the welfare of our patients. To this end, I propose another maxim for the leaders/mentors: "The unexamined life is not worth living."

Nuclear medicine began as a formal field of study after the Second World War, when the Atomic Energy Commission (AEC) placed an increasing emphasis on the peace dividend from technology developed in the national laboratories during the war. Initial training in medical applications of radioactivity was usually provided through courses sponsored by the AEC — most commonly at Oak Ridge National Laboratory, which is sponsored by the U.S. Department of Energy. In light of increasing interest in the field, the need for formalized training led to the formation of the American Board of Nuclear Medicine (ABNM), a conjoint board of Radiology, Internal Medicine, and Pathology, which issued its first certificates in 1972.

\section{HOW WE CAN ATTRACT YOUNG TRAINEES TO NUCLEAR MEDICINE?}

I have seen the field of nuclear medicine evolve dramatically since the earliest days before ${ }^{99 \mathrm{~m}} \mathrm{Tc}$ was widely available, when radiotracers such as colloidal gold ${ }^{198} \mathrm{Au}$ and ${ }^{203} \mathrm{Hg}$ were used to detect metastases to the liver and brain, respectively. The first scans I performed as a medical student used the rectilinear scanner, in which an organ, often the thyroid, was outlined in pointillist style on lightsensitive film. Today, there are hundreds of radiotracers that, at least in principle, can trace myriad metabolic pathways with tiny radioactive flashes that are converted to high-resolution functional images through 
the electronic wizardry of PET or SPECT alone or in combination with CT or MRI. Advances are moving at a startling pace, and this is a source of continuing wonder to me.

Young people choose training in a medical specialty that they believe will give them the greatest opportunity for a fulfilling and secure professional life. One thing I admire about nuclear medicine is our ability to self-renew. Throughout the history of the field, surges of talented newcomers have sought out training opportunities based on exciting new developments that have opened the door to scientific or clinical advances that address unmet medical needs. A recent example is the influx of skilled young radiologists entering our training programs, attracted in part by the promise of molecular imaging as a guide to precision medicine and the obvious excellence of fusion imaging, especially PET/CT and PET/MRI, as well as new radiotracers for in vivo biochemistry assessment. Individuals who are dual-boarded in Radiology and Nuclear Medicine will have a bright future in precision medicine and imaging.

From the beginning, talented individuals have transitioned to the nuclear medicine field early in their training or during midcareer from other specialties within medicine and a host of applied sciences. Thus, the field is continually revitalized-and transformed-by this diverse stream of nuclear medicine practitioners and basic scientists in radiochemistry, medical physics, and computer science. The migration of specialty interests to nuclear medicine was driven by opportunity and underlying medical need. Initially, nuclear medicine primarily comprised thyroidology, and hematology and many of the practitioners were endocrinologists or hematologists using radioiodine for diagnosis and therapy, especially ${ }^{131} \mathrm{I}$ and ${ }^{32} \mathrm{P}$. Then the focus shifted to the brain and neurochemicals, and neurologists joined the field, especially in research with PET and a variety of neurochemical and physiologic tracers such as ${ }^{15} \mathrm{O}$ and ${ }^{18} \mathrm{~F}$; next, it was cardiology, driven largely by cardiologists and internists who used ${ }^{99 \mathrm{~m}} \mathrm{Tc}$-labeled tracers. Radiochemists invented technetium-based cardiac drugs, then the priority shifted to higher-resolution PET imaging with coregistration through PET and CT, which brought in more newcomers to the field. And we are now at the beginning of another exciting change, perhaps the most exciting of all-the recognition that the power of the atom can cast light on the dark and diseased places in the body as well as restore health with curative radiation energy. We are already seeing a new group of medical oncologists and physician-scientists moving into the field of nuclear medicine to exploit its therapeutic potential.

To this point, I have emphasized the physician roles. But I know of no other specialty of medicine that involves such a tight integration with basic scientists and nonphysician scientists. This group occupies a variety of leadership and other key positions. After all, our specialty is based on the foundation of radioactive biochemicals and the tracer principle, requiring the expertise of chemists and radiochemists; electronic equipment to create images and measure radiation, requiring medical physicists and dosimetrists; and computer processing, requiring computer scientists and engineers. This multidisciplinary collaboration within nuclear medicine is a tremendously creative force.

\section{ADVICE TO EARLY-CAREER PROFESSIONALS}

My own training program consisted of $2 \mathrm{y}$ in internal medicine, $2 \mathrm{y}$ in nuclear medicine, and a 1-y research fellowship, totaling approximately $5 \mathrm{y}$. Please realize that training was less formalized then, before the ABNM was established. If personal pressures permit, $4 \mathrm{y}$ is a minimum target time, and an additional fellowship year is the minimum time in which to develop the skills needed to successfully pursue grant funding. I had the advantage of 2 outstanding role models/mentors (Drs. Nelp and Wagner, as previously mentioned), both of whom were internist/nuclear medicine physicians.

\section{PERSONAL BACKGROUND TO STIMULATE DISCUSSION}

I am a diplomate of that first examination in nuclear medicine. My certificate reads:

\section{"STEVEN MARK LARSON, M.D.}

HAS MET THE REQUIREMENTS OF THE BOARD, AND IS QUALIFIED TO PRACTICE AS A SPECIALIST IN ALL ASPECTS OF CLINICAL AND LABORATORY

\section{NUCLEAR MEDICINE}

INCLUDING BUT NOT LIMITED TO RADIOBIOASSAY, NUCLEAR IMAGING, IN VIVO MEASUREMENTS AND THERAPY WITH UNSEALED RADIONUCLIDES.

May 5, 1972"

For me, choosing a medical career was always an easy decision. I cannot recall an agonizing moment of self-doubt or a pang of regret. I decided to be a doctor while attending summer camp as a 12-y-old. It was a religious camp, and a medical missionary had just spoken and presented slides. I thought, I would like thathelping people, treating tropical diseases, doing what needed to be done-and from that point on, I never wavered in my choice.

I think a lot of people enter nuclear medicine because of the science, and how this interfaces with the world around us. This was certainly true for me. I was intrigued by the collateral physics, radiochemistry, radiotracer principles, kinetic modeling, and the history of development of artificial radioactivity and its biologic effects. Of course, radioactivity is simply one manifestation of the rhythmic energy of life-the sun and stars, fusion reactors, production of both stable and radioactive elements, matter of the universe, the elements made up of atoms (that are themselves tiny universes). Combine these inanimate wonders with biochemistry and the molecular mysteries of the human body-amazing! That is nuclear medicine.

My specific introduction to the science of radiation and radionuclides began as an undergraduate student at the University of Washington in Seattle (UW). I worked as a radiochemistry technician in a radiobiology laboratory monitoring plants and animals for radioactive fallout from atmospheric atom bomb testing. This experience instilled in me a lifelong interest in radiochemistry and radiation effects, and I honed my skills in inorganic and chelation chemistry. Once in medical school at UW, I gravitated to nuclear medicine, where I worked in the laboratory of Wil B. Nelp, MD, Chief of Nuclear Medicine at University Hospital, who taught me radiotracer experimental skills for biologic application. During medical school, I applied for and accepted a 15-mo National Institutes of Health (NIH)-sponsored fellowship, during which I developed the first successful "kit" formulation— ${ }^{99 m}$ Tc-sulfer colloid, a widely used radiopharmaceutical for lymphoscintigraphy and bone marrow imaging. After medical school, I continued on the path toward nuclear medicine through internal medicine. I found that I most enjoyed examining patients, discussing symptoms and reviewing lab tests, and ultimately solving the riddle of what went wrong and determining how we could fix it.

After training in internal medicine, I took the American Board of Internal Medicine examination in 1971. I successfully competed for a U.S. Public Health Service (USPHS) fellowship in nuclear medicine and was appointed as a second lieutenant at the $\mathrm{NIH}$ in Bethesda, Maryland. It was a great learning experience that consolidated the knowledge about nuclear medicine I had gained in the laboratory with strong general clinical nuclear medicine 
experience. I subsequently accepted my first faculty position as an Assistant Professor at Johns Hopkins Medical Institutions, where I developed skills in written presentation of basic and clinical science and grantsmanship under the direct tutelage of Dr. Henry N. Wagner, a giant in nuclear medicine (for more details and timelines, and to see what an academic career looks like, see the supplemental biosketch [supplemental materials are available at http://jnm.snmjournals.org]).

Ultimately, I became the Chief of Nuclear Medicine at Memorial Sloan Kettering Cancer Center in 1988 where, with the help of many physicians and scientists, we developed a state-of-the-art nuclear medicine program. The program included a therapy-oriented practice to treat patients with radioactive drugs and a thyroid clinic for patients with advanced cancers. In collaboration with the endocrinology group and others in nuclear medicine, we treated patients with highdose radioiodine and were able to cure some patients who would have otherwise had progressive tumors leading to death. We also established ${ }^{124} \mathrm{I}$ as an innovative tool for dosimetry and have since translated discoveries about reinduction of radioiodine therapy to expand the pool of responding patients. One of my patients established a support group for patients with advanced thyroid cancer called "The Light of Life," based on the idea of the life-giving energy (the light) that comes from effectively targeted radiation for thyroid cancer. I am currently the Chairman of the Board of this organization, which continues to provide helpful information to patients and recognizes leaders in thyroid cancer research and clinical care.

With regard to novel radiotherapeutics, I also collaborated with skilled immunologists, medical oncologists, and pediatric oncologists to develop improved clinical regimens for targeted radiotherapy based on radioimmunotherapy. These include the first $\alpha$-therapy radiolabeled antibodies administered to humans and novel antineuroblastoma antibodies designed to cure central nervous system recurrence. My laboratory continues to develop new drugs; among the 25 patents we hold, new agents and other advancements in pretargeted radioimmunotherapy are achieving cures.

In the end, I have had a successful academic career in nuclear medicine. This was the professional future that I had hoped for, without knowing its full extent when I began my training. Of course, I was lucky, without a doubt. But I was no different from any of today's qualified rising trainees in nuclear medicine. My advice is to set your goal along a training path that will fulfill your potential and offer a realistic chance of success. Also, an academic career like mine may not be the best fit for you, but there are plenty of rewarding opportunities in the field. Your career in nuclear medicine can be equally rewarding through performing diagnosis or therapy with radionuclides if you have the interest, motivation, and the right set of skills.

\section{A Sample of Some Specific Opportunities}

If your skill set is clinically oriented and you feel optimistic about a future that includes a mixture of radiology and nuclear medicine practice, if at all possible, be sure to have dual board certification in radiology and nuclear medicine. A practical pathway is the new 16-mo nuclear medicine option after a 3-y term in basic radiology, which you can complete as part of your 4-y commitment (usually a 4-mo stint in nuclear medicine is included in the basic 3-y radiology curriculum). Most individuals I have talked to with this background are comfortable with general nuclear medicine, the fundamentals of nuclear cardiology, and PET/CT interpretation after this period of training. This training, along with general radiology competency, is certainly an ample foundation for clinical practice. The drawback of this training program is a lack of time for research training; further, you will likely not feel comfortable with the management of patients receiving targeted radiotherapy. I suspect that this group, which is moving into the field in force, will represent a majority of U.S. practitioners over the next $10 \mathrm{y}$.

Some of you, for one reason or another, may need to shorten your training period as much as possible. Many students finish medical school with major debt and need to begin earning a salary immediately. In the standard radiology training program of $4 \mathrm{y}$, there are 4 mo of nuclear medicine training. Although it is hard to know the numbers, depending on the practice situation, many use that knowledge base in a diverse practice of radiology that involves nuclear medicine. Some in nuclear medicine are also anxious to finish training and after the 3 y required for the ABNM certificate, will be satisfied to enter private practice. I say go for it, but bear in mind that you run the risk of being displaced by others with greater training and expertise.

A growing facet of nuclear medicine is theranostics, the science of diagnosis combined with therapy. A theranostic is a drug with intrinsic diagnostic and therapeutic properties, for example, radiolabeled peptides such as ${ }^{177}$ Lu DOTATATE (Lutathera) or the iodine pair ${ }^{124} \mathrm{I} /{ }^{131} \mathrm{I}$. The number of these drugs entering nuclear medicine and treatment with internal emitters is increasing. Because of the therapy aspect, theranostics lends itself to an internal medicine or a medical oncology mindset and requires patient management skills.

\section{CAVEATS}

\section{The Weather Ahead: Storms or Sunny Skies? The Forecast: Both}

Why do bad things happen to good people? (Recommended further reading: Book of Job.)

I am a member for life of the ABNM, having served as the Director from 1998 to 2004 . I have trained more than 100 residents, fellows, and scientists in nuclear medicine methods, many of whom have gone on to become leaders of successful programs throughout the world. In recent times, I have observed that, at least in the United States, the graduates of our training programs with the brightest futures have pursued dual boards in nuclear medicine and radiology. I have witnessed unhappy times, when careers did not progress as expected and for one reason or another, a change was needed midcareer. Regardless, I never questioned that these individuals made a sound decision in choosing nuclear medicine as a medical focus.

I have observed disruptive changes impacting nuclear medicine physicians in midcareer whose paths were cut short or who had to enter a less desirable practice. These are good people; why do these bad things happen? Several them had come to the unfortunate realization that $\mathrm{ABNM}$ certification does not guarantee a smooth future. The cold reality is that the impersonal nature of marketplace capitalism sometimes leads to harsh and unexpected consequences. In the United States, nuclear medicine practice that is predominantly $\mathrm{PET} / \mathrm{CT}$ or fusion imaging is being pushed into the domain of individuals with dual boards (ABNM/ABR [American Board of Radiology]) because this is believed to provide the best broad training. There are exceptions to this rule, but as a trainee, why not follow such a path if this is your vision of a successful future practice? 


\section{Advice to Leaders/Mentors with Oversight of \\ Training Programs}

"An unexamined life is not worth living" (attributed to Socrates).

Several knowledgeable individuals have raised grave concerns about the economic health of the nuclear medicine field, particularly the relevance of current training programs to medical practice $(1,2)$. I believe these fears are unfounded, and that the combination of molecular imaging and theranostics is an unbeatable pairing with enormous growth potential (3). Responsible leaders know that if we want to optimize patient care, subspecialists must be nurtured in order to devote their careers to the areas of nuclear medicine best suited to their skill set. Most radiologists are not sufficiently trained in patient management, in my opinion, nor are they interested in the therapeutic aspects of nuclear medicine. Still, since patient management constitutes a significant part of therapy with radionuclides, the group most likely to succeed in this aspect of the field will be dually certified nuclear medicine/internal medicine physicians. On the other hand, radiologists appear to be very comfortable with cross-sectional imaging, CT, and MRI, especially in comparison to trainees specializing in only internal medicine or nuclear medicine. Nonetheless, both can learn if taught the appropriate techniques, and it is essential to fill in the gaps in our trainees' education.

The anxiety of individuals with nuclear medicine training alone is understandable, given the world we live in and the real-life examples of those who have been ousted for reasons not related to their professional conduct. I believe this relates in part to the hegemony of radiology in clinical and academic settings, and the fact that organized radiology is sufficiently strong to enforce policies favoring radiologists, if it so chooses. Most chairmen of radiology I know realize their responsibilities and do not fall into this trap; they understand their moral obligation to be objective and fair to nuclear medicine trainees as well as faculty members under their supervision.

Recently, a summit was convened to address the issue of training in nuclear medicine in the context of the U.S. medical system and the differences of opinion within professional groups. This is extremely important, and leaders from all sides should participate in and support this effort. The conflict we see among professional groups is not good for medicine as a whole and inhibits trainees from entering the field. As leaders, it is our solemn duty to resolve these issues amicably.

Four essential policies for the near future:

1. Continue to welcome radiology/nuclear medicine dual-boarded individuals with 16 mo of training in nuclear medicine within the $4 \mathrm{y}$ of their radiology training program.
2. Encourage medical oncologists/internists to enter the field through policies that provide a similar advantage to training over a 4-y period, as would be afforded by any other specialty.

3. Offer a training fellowship within a 4-y period that is focused on research, similar to the model of radiation oncology.

4. Actively support continuing medical education (CME) for trainees at every level so they can keep up with current research and best practices in the field.

Training programs should focus on 3 promising areas of study $(4,5)$ :

1. Clinical imaging for precision medicine with PET/CT and PET/ MRI.

2. Clinical theranostics, dosimetry, and treatment

3. Research fellowships (1-2 y in duration) to prepare trainees for academic careers, tailored to their individual interests.

\section{CONCLUSION AND A FINAL PERSONAL NOTE}

I entered nuclear medicine $60 \mathrm{y}$ ago when internists were common in the field and much of the leadership had dual certifications in nuclear medicine and internal medicine. I have had a great career, but times are changing with respect to opportunities, and some of these changes clearly track along lines of prior training. Quality of training is extremely important, and training programs must be tailored to an individual's skill set and desired career trajectory. In addition, as leaders, we must recognize our obligation to provide the same types of opportunities to trainees of all backgrounds, because diversity is essential for our field. Every trainee, regardless of sex, race, ethnicity, or any other characteristic, should have the same opportunity to succeed that I had when I entered the field. Leaders in the fields of radiology and nuclear medicine must ensure that a diverse set of trainees can take maximum advantage of the twin opportunities that the future holds-namely, (1) molecular imaging and precision medicine; and (2) radiotheranostics and therapy driven by parenteral administration of unsealed targeted radionuclides.

\section{REFERENCES}

1. Graham MM, Delbeke D, Jadvar H. Point: the existential threat to nuclear medicine. J Am Coll Radiol. 2018;15:384-386.

2. Guiberteau MJ, Oates ME. Counterpoint: nuclear medicine's decline: radiology is the solution, not the problem. $J$ Am Coll Radiol. 2018;15:387-389.

3. Segall GM, Pryma DA, Fair JR. A bright future for nuclear medicine. J Am Coll Radiol. 2019;16(4 pt A):531-532.

4. Delbeke D, Royal HD, Frey KA, Graham MM, Segall GM. SNMMI/ABNM joint position statement on optimizing training in nuclear medicine in the era of hybrid imaging. J Nucl Med. 2012;53:1490-1494.

5. Segall GM, Grady EE, Fair JR, Ghesani MV, Gordon L. Nuclear medicine training in the United States. J Nucl Med. 2017;58:1733-1734. 